\title{
Performance analysis of bio-Signal processing in ocean Environment using soft computing techniques
}

\author{
N. R. Krishnamoorthy, Immanuel Rajkumar, Jerry Alexander, D. Marshiana \\ Sathyabama Institute of Science and Technology, India
}

\begin{tabular}{l} 
Article Info \\
\hline Article history: \\
Received Mar 23, 2019 \\
Revised Dec 12, 2019 \\
Accepted Jan 8, 2020
\end{tabular}

Keywords:

Feed-forward neural network

Gradient

Hidden layer

Mean square error

Micro-organism

Watermark

\begin{abstract}
Wireless communication has become an essential technology in our day-to-day life both in air and water medium. To monitor the health parameter of human begins, advancement techniques like internet of things is evolved. But to analyze underwater living organisms health parameters, researchers finding difficulties to do so. The reason behind is underwater channels has drawbacks like signal degradation due to multipath propagation, severe ambient noise and Attenuation by bottom and surface loss. In this paper Artificial Neural Networks (ANN) is used to perform data transfer in water medium. A sample EEG signal is generated and trained with 2 and 20 hidden layers. Simulation result showed that error free communication is achieved with 20 hidden layers at 10th iteration. The proposed algorithm is validated using a real time watermark toolbox. Two different modulation scheme was applied along with ANN. In the first scenario, the EEG signal is modulated using convolution code and decoded by Viterbi Algorithm. Multiplexing technique is applied in the second scenario. It is observed that energy level in the order of $40 \mathrm{~dB}$ is required for least error rate. It is also evident from simulation result that maximum of $5 \% \mathrm{CP}$ can be maintained to attain the least Mean Square Error.
\end{abstract}

Copyright @ 2020 Institute of Advanced Engineering and Science. All rights reserved.

Corresponding Author:

N. R. Krishnamoorthy,

Department of Electrical and Instrumentation Engineering,

Sathyabama Institute of Science and Technology,

Rajiv Gandhi Salai, Sholinganallur, Chennai, Tamilnadu, India.

Email: krishnamoorthy.eni@sathyabama.ac.in

\section{INTRODUCTION}

Wireless communication has become an essential technology in our day-to-day life in under and above the ground level. But there is difference in data transmission in underwater and air medium. Data transmission in underwater can be done by acoustic waves rather than Electromagnetic waves [1], since it lacks in the long range propagation. Underwater communication has few drawbacks [2,3] compared to air medium such as multipath propagation [4], Attenuation factor, Signal losses [5] and Limited Bandwidth. Researchers [6] showed that the bandwidth of an underwater channel is directly proportional to the transmission distance and its achievable data rate is $1 \mathrm{kbps}$ for long range $(50 \mathrm{kms})$ and $10 \mathrm{kbps}$ for short range of few kms [7]. In [8, 9], author designed an optical power splitter for underwater wireless communication application. A microstructure $\mathrm{GaN}$ semiconductor was developed by Metal-organics Chemical Deposition method. The result showed imbalance loss of $0.17 \mathrm{~dB}$.

In [10], underwater bidirectional communication using LED was carried out by the authors. Voltage level of 3.46 volts is required for the transmission of data from transmitter to receiver. On another side, a 4.2 volts is needed for receiver to capture the data. Using such a voltage level will cause harmful to the living organism, hence researchers can avoid using LED source and go for acoustic signal as a communication purpose. Lot of modern coding techniques are applied for underwater communication. A Reliability Level List based decoding algorithm [11] is applied to an AWGN channel and attained an BER in the order of 10-4. 
The algorithm was tested on Single Input Singe Output (SISO) channel alone. To enhance the bandwidth utilization of the receiver, researchers will make use of Rake receiver.

In [12], author used Continuous Wavelet Transform along with Rake receiver. It was tested for both line of sight and non-line of sight channels with different $\mathrm{dB}$ levels. The distance between the transmitter and receiver maintained is about 4 meters results in the BER in the order of 10-3. MIMO-OFDM system was implemented by the author for Rayleigh and Rician Channel [13]. Encoding was done by OFDM technique [14-16] with CP and in the receiver section LMS and RLS algorithm was developed and error rate is calculated. For application like ocean pollution monitoring, industrial sensing and disaster prevention purpose, an Underwater Acoustic Sensor Networks [17] was designed. RF signal along with Zigbee was used for point to point communication. Other techniques like turbo equalization [18], Low Density Parity Check Codes [19], Space Frequency Block coding [20] and Decision Feedback Equalizer [21, 22] are applied to the underwater channel.

In the field of science and industry, modernizing is carried out by means of sensor networks [23] to monitor the industrial applications [24], micro habitat [25] and underwater environmental system [26]. To locate and rescue the victims in the sea ocean, underwater robots [27] can be used. The advantage of using robots is it can dive and swim even at deep sea where humans cannot do and brings safety to the human life. Robots coordinate among themselves to solve a certain problem. While doing so, it is very important to see how effective the communication is carried out. The data transmission in underwater is very difficult due to its strong relative motion of the robots. In this paper, soft computing techniques such as Feed-Forward Neural Network is applied to study the performance of the underwater channel. Sample bio EEG signal is generated and communicated from one end to other end using neural network.

\section{RESEARCH METHOD}

To mimic the human nervous system and operation of brain, an Artificial Neural Network(ANN) is invented. For varieties of Artificial Intelligence operation like data prediction, pattern recognition and clustering, ANN are used intensively. The basic element in ANN is processing element called as neurons which act similar to the neural cells in human brain. Subgroup of these neurons are referred as layer.ANN comprises of one input and output layer with one or more than one hidden layers. Neurons will receive the signal either from another neuron or any other external environment and it is passed through a series of neurons and finally to the output layer. In the training stage, a set of input and output pairs are presented to the network. The network is excited with input, the weights of the each neurons are modified and an optimal value are obtained. After that, actual input data is allowed to pass through the network and output data are computed with that fixed weights of neurons. The translation of signals from input to the output response is referred as transfer function of the processing elements. The underwater channel is modeled using mirror image technique. The attenuation and noise of the ocean is calculated using Wenz Curve and ambient noise formula. Along with this factor the signal degradation by multipath propagation is also considered. The neural network is trained with the four different set of impulse response of the channel. A sample EEG signal is generated using MATLAB software and the performance analysis of ANN is determined.

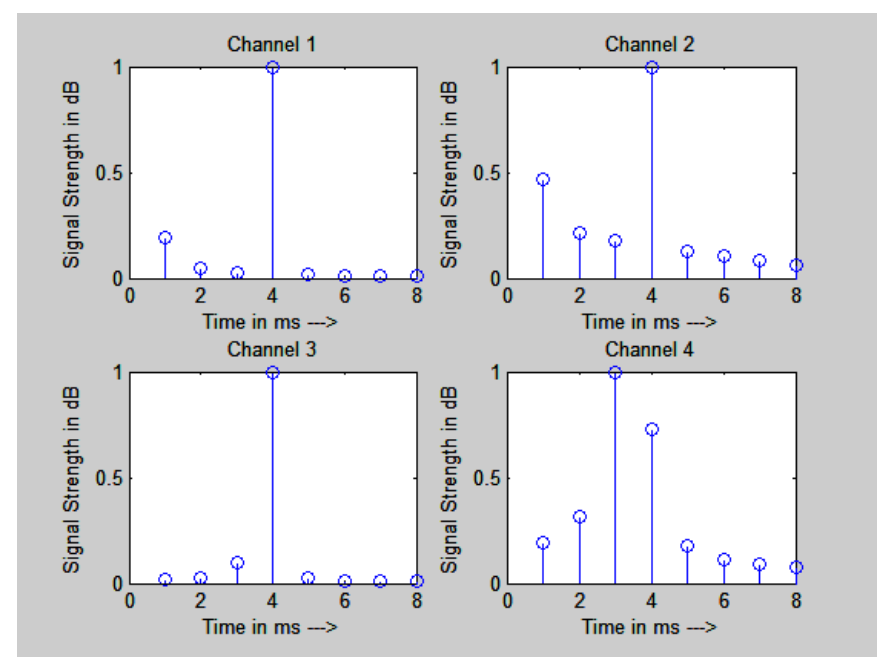

Figure 1. Impulse response of the channels 


\section{RESULTS AND ANALYSIS}

For the simulation purpose, four different ocean condition are assumed. First one is short distance, where the source and destination are separated by short distance of 500 meters with the ocean depth of $1 \mathrm{~km}$, second condition is same as first one with slight additional condition that sender and receiver will have a drift of $1 \mathrm{~m} / \mathrm{s}$. The third and fourth condition have a long distance of $10 \mathrm{~km}$ with and without drift respectively. These four different conditions are named as four channel for the evaluation purpose. The channel impulse response are shown in the Figure 1. The attenuation factor, Ambient noise and surface-bottom loss are calculated according to the condition and optimal frequency is calculated as $400 \mathrm{~Hz}$ for channel 1 and 2 and $100 \mathrm{~Hz}$ for chennal 3 and 4. The sample EEG signal is generated using this optimal frequency and sent to these four different conditions using feed-forward neural network.

It is found from the Figure 2 that, for a 2 hidden layer architecture a minimum Mean Square Error (MSE) of 0.05 for channel 4 and maximum MSE of 0.15 for the channel1. Figure 2 also shows the MSE of the four channels for 20 hidden layer architecture, We obtained MSE level of almost zero for all the channels with data Energy level (Eb/No) of $10 \mathrm{~dB}$ with increased iteration values. It is also observed from the simulation that the gradient value of the neural network is start decreasing from 0.45 to $10-5$ with Eb/No value of 5 and corresponding $\sigma$ value also decreases from 0.01 to $10-5$ with Eb/No value of 5 . It is evident that the Signals are received back without any defect using feed-forward network with 20 neurons in the hidden layer. The drawback in it is that the iteration level is very high. To overcome these, it is concluded that one can try the adaptive neural network in the channel to compensate the Doppler shift of the underwater acoustic channel.
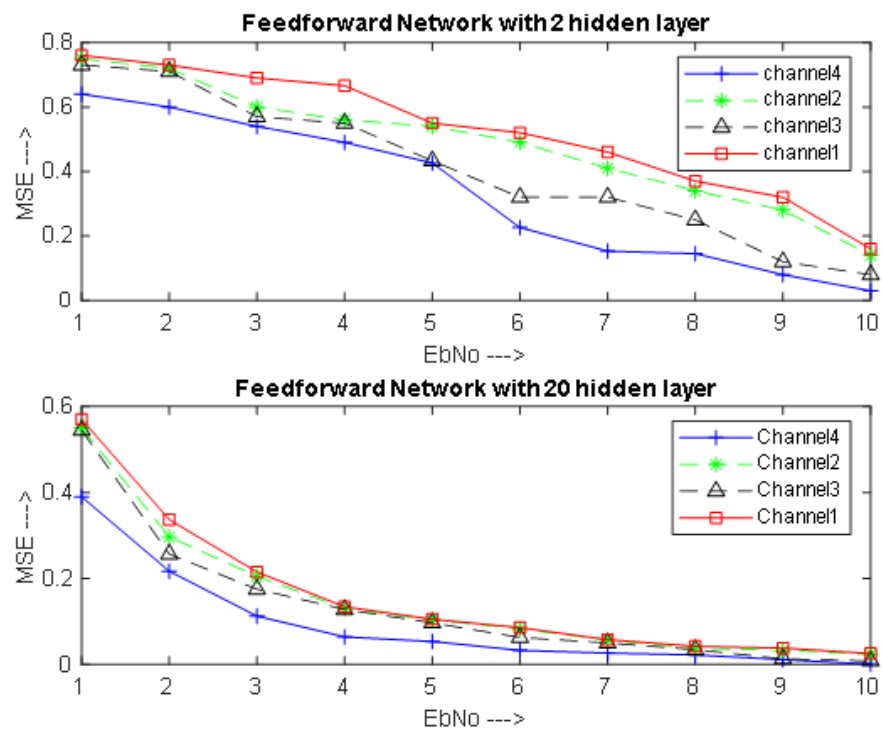

Figure 2. Performance of four channel using feed-forward neural network with 2 and 20 neurons in hidden layer

\section{VALIDATION OF PROPOSED ALGORITHM}

During the past decades, numerous coding techniques has been developed for data transmission in water medium. Unlike air medium, it is very difficult to validate the algorithm in underwater communication. In 2016, underwater AcousTic channel Replay benchMARK (WATERMARK) [28, 29] is introduced for underwater communication researcher. It is a realistic simulation toolbox which enables the researcher to develop, test and compare their algorithm in real time environment. The toolbox consists of five different channels corresponds to different environmental condition. The channels are named in the favor of the location as Norway-Oslofjord (NOF1), Norway-Continental Shelf (NCS1), Brest Commercial Harbour (BCH1), Kauai Acomms MURI 2011 (KAM11) experiment (KAU1) and KAU2. Depending upon the number of input and output channels, models are categorize as either Single Input Single Output (SISO) or Single Input Multipe Output (SIMO). Table 1 shows the environmental condition of the five channels. The various parameters considered in the watermark toolbox are range, type of transmitter and receiver deployment, frequency band, Doppler coverage etc. In this paper, NOF1 channel is considered to validate the proposed algorithm. 

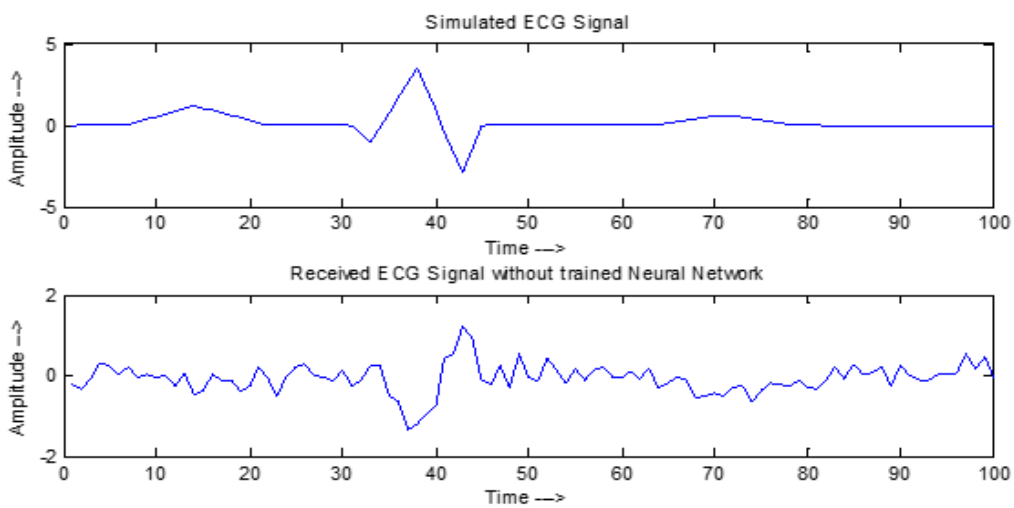

Received ECG Signal with trained Neural Network

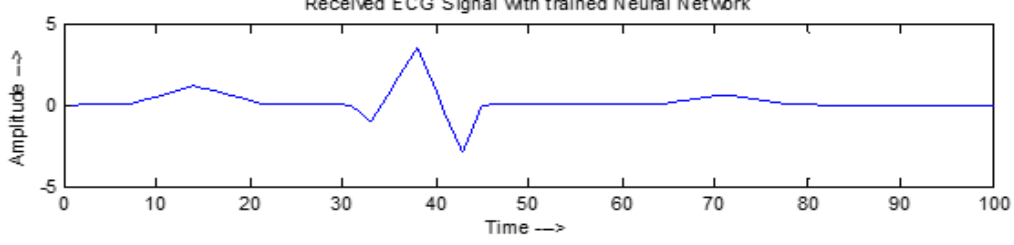

Figure 3. Retrieval of sample ECG from the UAC using feed-forward neural network

AEEG signal is generated and modulated using BPSK. Then it is allowed to pass through the NOF1 channel. For validation of ANN in underwater channel, two different coding technique is employed. In the first scenario, the modulated data is encoded using convolution code and decoded by Viterbi Algorithm. Multiplexing technique is applied in the second scenario. In the multiplexed technique, the modulated data is converted into time domain and send it to channel as a frames. To avoid the effect of the inter-symbol interference of the channel a portion of encoded data is added before the each frame. This process is referred as Cyclic Prefix (CP). The length of the CP is varied at three different level as 5\%, $15 \%$ and $25 \%$ with respect to the modulated data. For example, if the no of bits in a single frame is 20 , then $\mathrm{CP}$ length as 1, 3 and 5 bits. Table 2 to 5 shows the MSE of the NOF1 channel using convolution code and Multiplexing technique. Table 2 shows the MSE of the NOF1 channel using convolution code. The data is encoded using six different generator polynomials and decoded using Viterbi algorithm. It is observed that the MSE value is decreases with less polynomial complexity. The effects of variation of CP length in multiplexing technique is studied and summarized from Table 3 to 5 . In this scenario, increase in $\mathrm{CP}$ value results with more MSE. It is concluded that to obtain the better MSE, the ANN network is applied along with multiplexing technique with less CP value.

Table 1. Environmental condition of the channels in watermark toolbox

\begin{tabular}{cccccc}
\hline Name & NOF1 & NCS1 & BCH1 & KAU1 & KAU2 \\
\hline Environment & Fjord & Shelf & Harbor & Shelf & Shelf \\
Time of Year & June & June & May & July & $1080 \mathrm{~m}$ \\
Range & $750 \mathrm{~m}$ & $540 \mathrm{~m}$ & $800 \mathrm{~m}$ & $3160 \mathrm{~m}$ \\
Water Depth & $10 \mathrm{~m}$ & $80 \mathrm{~m}$ & $20 \mathrm{~m}$ & $100 \mathrm{~m}$ & $100 \mathrm{~m}$ \\
Transmitter Deployment & Bottom & Bottom & Suspended & Towed & Towed \\
Receiver Deployment & Bottom & Bottom & Suspended & Suspended & Suspended \\
Probe Signal type & LFM Train & Pseudo-noise & Pseudo-noise & LFM Train & LFM Train \\
Frequency Band & $10-18 \mathrm{kHz}$ & $10-18 \mathrm{kHz}$ & $32.5-37.5 \mathrm{kHz}$ & $4-8 \mathrm{kHz}$ & $4-8 \mathrm{kHz}$ \\
Doppler coverage & $7.8 \mathrm{~Hz}$ & $31.4 \mathrm{~Hz}$ & $59.4 \mathrm{~Hz}$ & $7.8 \mathrm{~Hz}$ & $32.9 \mathrm{~Hz}$ \\
Type & SISO & SISO & SIMO & SIMO & SIMO \\
\hline
\end{tabular}

Table 2. MSE of channel using convolution code

\begin{tabular}{ccccccccccc}
\hline \multirow{2}{*}{ Polynomial } & \multicolumn{10}{c}{ Signal Strength in dB } \\
\cline { 2 - 11 } & 5 & 10 & 15 & 20 & 25 & 30 & 35 & 40 & 45 & 50 \\
\hline$(1,3)$ & 0.526 & 0.526 & 0.526 & 0.526 & 0.512 & 0.456 & 0.298 & 0.140 & 0.070 & 0 \\
$(1,5)$ & 0.544 & 0.544 & 0.544 & 0.544 & 0.392 & 0.215 & 0.125 & 0.035 & 0.017 & 0 \\
$(10,11)$ & 0.544 & 0.544 & 0.544 & 0.544 & 0.456 & 0.368 & 0.184 & 0 & 0 & 0 \\
$(1,2,3)$ & 0.544 & 0.544 & 0.544 & 0.544 & 0.454 & 0.421 & 0.210 & 0.010 & 0.001 & 0 \\
$(1,4,5)$ & 0.544 & 0.544 & 0.544 & 0.544 & 0.443 & 0.310 & 0.193 & 0 & 0 & 0 \\
$(10,11,14)$ & 0.544 & 0.544 & 0.544 & 0.520 & 0.520 & 0.430 & 0.193 & 0.087 & 0 & 0 \\
\hline
\end{tabular}


Table 3. MSE of channel using multiplexing technique with $5 \%$ cyclic prefix

\begin{tabular}{|c|c|c|c|c|c|c|c|c|c|c|}
\hline \multirow{2}{*}{ Polynomial } & \multicolumn{10}{|c|}{ Eb/NoValue } \\
\hline & 5 & 10 & 15 & 20 & 25 & 30 & 35 & 40 & 45 & 50 \\
\hline$(1,3)$ & 0.526 & 0.509 & 0.501 & 0.491 & 0.420 & 0.333 & 0.193 & 0 & 0 & 0 \\
\hline$(1,5)$ & 0.474 & 0.439 & 0.421 & 0.403 & 0.386 & 0.351 & 0.035 & 0 & 0 & 0 \\
\hline$(10,11)$ & 0.544 & 0.491 & 0.438 & 0.421 & 0.386 & 0.230 & 0.088 & 0.017 & 0 & 0 \\
\hline$(1,2,3)$ & 0.596 & 0.579 & 0.520 & 0.500 & 0.351 & 0.123 & 0.017 & 0 & 0 & 0 \\
\hline$(1,4,5)$ & 0.526 & 0.544 & 0.526 & 0.526 & 0.360 & 0.017 & 0 & 0 & 0 & 0 \\
\hline$(10,11,14)$ & 0.491 & 0.474 & 0.474 & 0.474 & 0.316 & 0 & 0 & 0 & 0 & 0 \\
\hline
\end{tabular}

Table 4. MSE of channel using multiplexing technique with $15 \%$ cyclic prefix

\begin{tabular}{|c|c|c|c|c|c|c|c|c|c|c|}
\hline \multirow[t]{2}{*}{ Polynomial } & \multicolumn{10}{|c|}{ Eb/NoValue } \\
\hline & 5 & 10 & 15 & 20 & 25 & 30 & 35 & 40 & 45 & 50 \\
\hline$(1,3)$ & 0.508 & 0.509 & 0.491 & 0.465 & 0.405 & 0.281 & 0.158 & 0.035 & 0 & 0 \\
\hline$(1,5)$ & 0.508 & 0.491 & 0.474 & 0.474 & 0.439 & 0.254 & 0.052 & 0.017 & 0.017 & 0 \\
\hline$(10,11)$ & 0.491 & 0.491 & 0.474 & 0.456 & 0.410 & 0.316 & 0.228 & 0.053 & 0.017 & 0.017 \\
\hline$(1,2,3)$ & 0.491 & 0.491 & 0.491 & 0.473 & 0.474 & 0.421 & 0.228 & 0.053 & 0.053 & 0.053 \\
\hline$(1,4,5)$ & 0.491 & 0.474 & 0.474 & 0.456 & 0.400 & 0.320 & 0.035 & 0.035 & 0 & 0 \\
\hline$(10,11,14)$ & 0.579 & 0.550 & 0.544 & 0.520 & 0.520 & 0.298 & 0.088 & 0.017 & 0.017 & 0 \\
\hline
\end{tabular}

Table 5. MSE of channel using multiplexing technique with $25 \%$ cyclic prefix

\begin{tabular}{|c|c|c|c|c|c|c|c|c|c|c|}
\hline \multirow[t]{2}{*}{ Polynomial } & \multicolumn{10}{|c|}{ Eb/NoValue } \\
\hline & 5 & 10 & 15 & 20 & 25 & 30 & 35 & 40 & 45 & 50 \\
\hline$(1,3)$ & 0.474 & 0.456 & 0.438 & 0.435 & 0.421 & 0.333 & 0.193 & 0 & 0 & 0 \\
\hline$(1,5)$ & 0.474 & 0.438 & 0.421 & 0.421 & 0.403 & 0.351 & 0.035 & 0 & 0 & 0 \\
\hline$(10,11)$ & 0.546 & 0.491 & 0.438 & 0.421 & 0.386 & 0.215 & 0.087 & 0.017 & 0 & 0 \\
\hline$(1,2,3)$ & 0.526 & 0.508 & 0.491 & 0.491 & 0.456 & 0.210 & 0.158 & 0.140 & 0.105 & 0.088 \\
\hline$(1,4,5)$ & 0.561 & 0.544 & 0.544 & 0.491 & 0.250 & 0.150 & 0.088 & 0.088 & 0.088 & 0.088 \\
\hline$(10,11,14)$ & 0.561 & 0.523 & 0.523 & 0.508 & 0.263 & 0.123 & 0.123 & 0.070 & 0.070 & 0.060 \\
\hline
\end{tabular}

\section{CONCLUSION}

The generated EEG waveforms is retrieved back successfully using ANN in underwater channel. It is concluded from the result that the ANN performs well and gives least error rate as the iteration level and number of hidden layers increases. The proposed algorithm is validated using watermark toolbox. It is observed that the for standard algorithms like convolution and Multiplexing techniques, energy level in the order of $40 \mathrm{~dB}$ is required. For multiplexing techniques, as the length of the CP increases, error rate also increases. It is evodent that maximum of 5\% CP can be maintained to attain the least MSE. Researcher can adopt the ANN technique along with the multiplexing and analysis the performance of the ANN in underwater channel.

\section{REFERENCES}

[1] Abdou. A. A., et al., "Electromagnetic (EM) Wave Propagation for the Development of An Underwater Wireless Sensor Network (WSN)," SENSORS, 2011 IEEE, pp. 1571-1574, 2011.

[2] Liu. L., Zhou. S., and Cui. J.-H., "Prospects and Problems of Wireless Communication for Underwater Sensor Networks," Wireless Communications \& Mobile Computing, vol. 8, no. 8, pp. 977-994, 2008.

[3] Yu Luo., Lina Pu., Michael. Z., and Zheng Peng Jun-Hong Cui., "Challenges and Opportunities of Underwater Cognitive Acoustic Networks," IEEE Transactions on Emerging Topics in Computing, vol. 2, no. 2, pp. 198-211, 2014.

[4] Sanchez. A., Blanc. S., Yuste. P., and Serrano. J. A., "Low Cost and High Efficient Acoustic Modem for Underwater Sensor Networks," OCEANS, 2011 IEEE, Spain, pp. 1-10, 2011.

[5] Stojanovic. M., "Underwater Wireless Communications: Current Achievements and Research Challenges," IEEE Oceanic Engineering Society Newsletter, pp. 1-5, 2006.

[6] Cui. J.-H., Kong. J., Gerla. M., and Zhou. S. "The Challenges of Building Mobile Underwater Wireless Networks for Aquatic Applications," Network, IEEE, vol. 20, no. 3, pp. 12-18, 2006.

[7] Qin Lu., Yi Huang., and Zhaohui W. S. Z., "Characterization and Receiver Design for Underwater Acoustic Channels with Large Doppler Spread", OCEANS - MTS/IEEE Washington, pp. 1-6, 2015.

[8] Retno. W., P. Nyi. R., and P. Elhadj. D., "III-Nitride Semiconductors based Optical Power Splitter Device Design for Underwater Application," International Journal of Electrical and Computer Engineering, vol. 8, no. 5, pp. 3866-3874, 2018.

[9] Makoto. O., Tadashi. E., Koichi. M., and Naoto. M., "Wireless Power and Data Transfer System for Station-Based Autonomous Underwater Vehicles,” OCEANS - MTS/IEEE Washington, pp.1-5, 2015. 
[10] Arsyad. R. D., Andre. W., Muhamad R. A., "Bidirectional Underwater Visible Light Communication," International Journal of Electrical and Computer Engineering, vol. 8, no. 6, pp. 5203-5214, 2018.

[11] Sudharsan. V., Vijay. Karthik. V., and Yamuna. B., "Reliability Level List Based Iterative SISO Decoding Algorithm for Block Turbo Codes," TELKOMNIKA, vol. 16, no. 5, pp. 2040-2047, 2018

[12] Ch Navitha, Sivani. K., and Ashoka. Reddy, K., "Performance Evaluation of Adaptive Continuous Wavelet Transform based Rake Receiver for UWB Systems," International Journal of Electrical and Computer Engineering, vol. 8, no. 5, pp. 3444-3452, 2018.

[13] Srinu. P , K. Padma. R., K., and Subrahmanyam. N. B., "Performance Analysis of Adaptive Filter Channel Estimated MIMO OFDM Communication System," International Journal of Electrical and Computer Engineering, vol. 8, No. 5, pp. 3829-3838, 2018.

[14] Yuriy. V., Zakharov., Andrey. And K. Morozov., "OFDM Transmission Without Guard Interval in Fast-Varying Underwater Acoustic Channels," IEEE Journal of Oceanic Engineering, vol. 40, no. 1, pp. 144-158, 2015.

[15] Yashar. M. Aval., and Milica. Stojanovic., "Differentially Coherent Multichannel Detection of Acoustic OFDM Signals," IEEE Journal of Oceanic Engineering, vol. 40, no. 2, pp. 251-268, 2015.

[16] Lei Wan., et al., "Adaptive Modulation and Coding for Underwater Acoustic OFDM," IEEE Journal of Oceanic Engineering, vol. 40, no. 2, pp. 327-336, 2015.

[17] Youngjun. J. and Sangsoon. L., "Design and Implementation of Heterogeneous Surface Gateway for Underwater Acoustic Sensor Network," International Journal of Electrical and Computer Engineering, vol. 9, no. 2, pp. 1226-1231, 2019.

[18] Yanbo Wu., Min Zhu., and Xinguo Lit., "Sparse Linear Equalizers for Turbo Equalizations in Underwater Acoustic Communication," OCEANS - MTS/IEEE Washington, pp.1-6, 2015.

[19] Shunde. Z., Xin. Z., and Xiaoji. Z., "Iterative Frequency Domain Equalization Combined with LDPC Decoding for Single-Carrier Underwater Acoustic Communications," OCEANS MTS-IEEE Montereyon, pp. 1-5, 2016.

[20] Eduard. V. Z., and Milica. S., "Space-Frequency Block Coding for Underwater Acoustic Communications," IEEE Journal of Oceanic Engineering, vol. 40, no. 2, pp. 303-314, 2015.

[21] Xiaohui. Z., Fangjiong. C., FeiJi., and Hua. Y., "Variable Step Size Least Symbol Error Rate Adaptive Decision Feedback Turbo Equalization for Underwater Channel," OCEANS-MTS/IEEE Washington, pp. 1-4, 2016.

[22] Roee. D., and Lutz. L., "Adaptive Error-Correction Coding Scheme for Underwater Acoustic Communication Networks," IEEE Journal of Oceanic Engineering, vol. 40, no. 1, pp. 104-114, 2015.

[23] Heidemann. J., Wei. Y, Wills. J., Syed. A., and Yuan. L, "Research challenges and Applications for Underwater Sensor Networking," IEEE Wireless Communications and Networking Conference, pp. 228-235, 2006.

[24] Ramanathan. N., et al., "A Stream-Oriented Power Management Protocol for Low Duty Cycle Sensor Network Applications," In Proceedings of the IEEE Workshop on Embedded Networked Sensors, 2005.

[25] Cerpa. A., et al., "Monitoring: Application Driver for Wireless Communications Technology," In Proceedings of the ACM SIGCOMM Workshop on Data Communications in Latin America and the Caribbean, 2001.

[26] Kim. J. E., et al., "Adaptive MAC Scheme using Queue Information for Underwater Environmental Monitoring System," pp. 884-889, 2013.

[27] WeiXing. F., KeJun. W., XiuFen. Y., and ShuXiang. G,. "Novel Algorithms for Coordination of Underwater Swarm Robotics," pp. 654-659, 2006.

[28] Van Walree. P. A., Jenserud. T. and Smedsrud. M., "A Discrete-Time Channel Simulator Driven by Measured Scattering Functions,” IEEE Journal. Sel. Areas Commun., vol. 26, no. 9, pp.1628-1637, 2008.

[29] Van Walree P. A., "Propagation and Scattering Effects in Underwater Acoustic Communication Channels," IEEE Journal of Ocean Engineering, vol. 38, No. 4, pp. 614-631, 2013.

\section{BIOGRAPHIES OF AUTHORS}

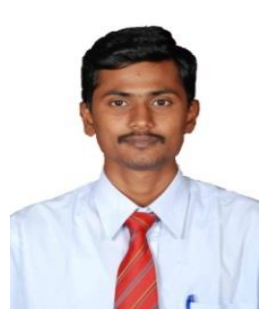

N. R. Krishnamoorthy, he graduated from Jerusalem College of Engineering, Chennai, affiliated to Madras University in 2004 with Bachelor's Degree in Electronics and Communication Engineering. Later he completed his M.E degree in Electronics and Control from Sathyabama Institute of Science and Technology, Chennai in 2007. He has joined as Lecturer in Sathyabama Institute of Science and Technology in 2008. His passion towards teaching made as Associate professor in the department of Electronics and Instrumentation Engineering in Sathyabama Institute of Science and Technology, Chennai. He played vital role in the sathyabamasat nanosatellite project as the payload subsystem incharge. He acquired a teaching experience of more than 13 years. His areas of interest include signal processing, wireless communication, coding techniques and integrated circuits. He attended various workshops conducted by the industrials. He guided several student projects and he made research publications in diverse Journals and Conferences. He is a life member of Indian Society of Technical Education. (ISTE). 


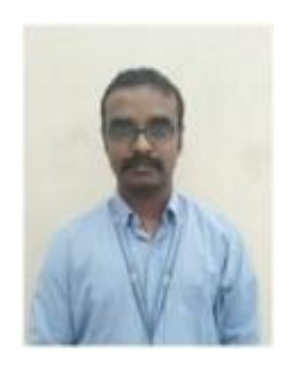

Immanuel Rajkumar, he graduated from Karunya Institute of Technology, Coimbatore affiliated to Madras University in 2002 with Electronics and Instrumentation Engineering. Later he completed his post graduation degree in Electronics \& Control Engineering from Sathyabama University , Chennai in 2004. He is currently doing his research in Faculty of Electronics \& Control Engineering at Sathyabama Institute of Science \& Technology, Chennai. His Area of interest includes is Agent based Technology, Industrial Instrumentation, Real time Embedded systems, Process Control.

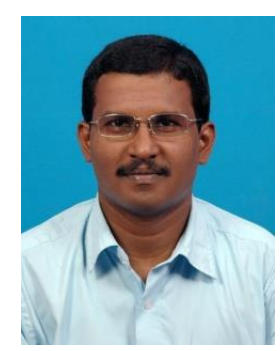

Jerry Alexander, he graduated from Noorul Islam College of Engineering affiliated to ManonmaniamSundaranar University in 1999 with Bachelor's Degree in Electrical and ElectronicsEngineering. Later he completed his M.E degree in Electronics and Control from Sathyabama Institute of Science and Technology, Chennai in 2004. He has joined as Lecturer in Sathyabama Institute of Science and Technology in 2004and .At the current outset he is an Assistant Professor in the department of Electronics and Communication Engineering at Sathyabama Institute of Science and Technology, Chennai. He has the teaching experience of more than $14 \frac{1}{2}$ years and about $2 \frac{1}{2}$ years in Industry. His areas of interest include signal processing and industrial automation. He attended various workshops \& conferences conducted by reputed organizations.He made research publications in Journals and Conferences.He is a life member of International Association of ENGineers.

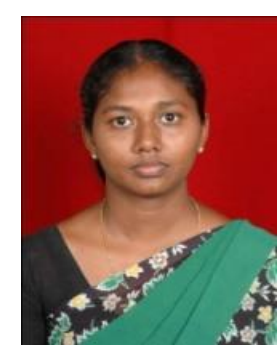

Marshiana D., completed her Ph.D. at Sathyabama University. Her areas of interest include Process control, controller design and soft computing techniques with 15 Publications. She is currently working as Assistant Professor and having teaching experience of 13 years. Her areas of interest include control system and process control, Soft computing techniques and control algorithms. She attended various workshops conducted by the industrials. She guided several student projects and she made research publications in diverse Journals and Conferences. 